\title{
Placements à long terme en villages d'enfants depuis plus de 30 ans : Troubles psychiques et prises en charge
}

\author{
Thirty years of long-term placement in children's villages: \\ psychological disorders and care
}

\author{
A.C. Dumaret, M. Crost \\ CERMES,-INSERM U750, 7 rue Guy Môquet, 94801 Villejuif cedex, France
}

\author{
* Auteur correspondant \\ Adresse e-mail : dumaret@vjf.cnrs.fr \\ Psychologue, ingénieur de recherche à l'INSERM
}

\begin{abstract}
Résumé en français
Les villages d'enfants ont pour spécificité d'accueillir des fratries sur un long terme. Cet article explore, sur une période de trente années, les difficultés psychologiques et comportementales des enfants et adolescents à travers des prises en charge qui ont évolué dans le temps. Des analyses multivariées mettent en évidence, pour l'ensemble de ces jeunes, l'importance de certaines pathologies parentales et de la gravité des carences et maltraitances sur les troubles psychiques. Les accompagnements et les suivis se sont développés et diversifiés entre les décennies 1960 et 1990 : à peine un cinquième des plus anciens ont eu au moins une prise en charge régulière $v s$ les quatre cinquièmes de ceux admis dans la période la plus récente. Ce placement de longue durée et les prises en charge effectuées ont permis une nette amélioration sur le plan psychologique et comportemental. Néanmoins, le fait que de nombreux jeunes présentant des troubles graves n'ont pas eu de soins appropriés soulève la question toujours actuelle de l'évaluation clinique non seulement au moment de l'admission mais aussi eu cours de placement.
\end{abstract}

Mots clé : protection de l'enfance, troubles psychologiques, comportement, scolarité Villages d'enfants, prise en charge, facteurs de risque

\section{Summary}

SOS Children's Villages specialise in welcoming siblings together over long periods. This article explores, over the course of thirty years, the psychological and behavioural difficulties of children and adolescents through the length of care practices that have evolved over time. For all these youths, multivaried analyses highlight the significance of certain parental pathologies and of the severity of neglect and maltreatment in the development of psychological disorders. Guidance and care practices were greatly expanded and diversified in the decades between 1960 and 1990: barely one-fifth of the oldest youths was regularly looked after, versus four-fifths of those admitted in later years. The long-term placements and care provided allowed clear improvements on both a psychological and behavioural level. However, the fact that numerous youths suffering from serious disorders did not receive appropriate care begs the still-relevant question of clinical evaluation, not only at the time of admission but also during placement. 
Keywords: child welfare, out-of-home care, siblings, behaviour, psychological problems, education, risk factors 


\section{Introduction}

Dans le domaine de la Protection de l'enfance, plusieurs écrits ont souligné le manque d'évaluations institutionnelles relatives aux décisions concernant les enfants et ont soulevé la question de l'adéquation des prises en charges à leurs besoins spécifiques [1, 2]. L'acuité d'une telle préoccupation évaluative, visible à travers de nombreux débats et interventions, s'est récemment traduite dans la loi du 2 janvier 2002 qui rénove l'action sociale et médico-sociale [3, 4 ]. Certaines institutions ont fait appel à des équipes de recherche afin de mieux comprendre leur fonctionnement et connaitre le devenir des enfants et adolescents pris en charge. Ainsi, une étude évaluative a récemment été menée sur le devenir à long terme des jeunes ayant vécu avec leur fratrie dans un village d'enfants (VE), plus de la moitié d'entre eux étant sortis depuis plus de vingt ans.

L'institution SOS Villages d'Enfants s'intéressait aux répercussions générales des modes de prises en charge sur les enfants et adolescents devenus des adultes et à la manière dont ont évolué les relations fraternelles. L'équipe de recherche se posait aussi la question des effets des carences graves et des maltraitances sur l'insertion générale à l'âge adulte après un placement stable et continu.

Dans ce présent article, nous nous centrerons sur la problématique du placement: l'évolution des accueils et des prises en charge pendant trois décennies d'une part, l'impact des facteurs de risque familiaux et individuels sur les difficultés psychiques des jeunes d'autre part.

\section{Le contexte institutionnel}


Les villages d'enfants (VE), maisons d'enfants à caractère social, sont des structures intermédiaires entre familles d'accueil et établissements, et ont pour spécificité l'accueil de fratries sur une longue durée. L'un d'eux, terrain de la recherche, se compose de dix maisons familiales. Ce village d'enfants, créé il y a un peu plus de trente ans, a vu son équipe psycho-éducative se structurer progressivement dans le milieu des années 1980 et se dessiner de nouveaux profils d'enfants accueillis (fratries non orphelines, sorties ou réorientations avant l'âge de la majorité). L'accueil s'est professionnalisé, la question des relations parents-enfants a fait l'objet de réflexions et a donné lieu à un encadrement des visites. C'est en janvier 2005 que le statut professionnel des mères SOS sera reconnu : elles deviennent alors des éducatrices familiales.

\section{Matériel et méthode}

La méthodologie utilisée, une approche longitudinale dans le passé [5], a nécessité plus de trois années de travail sur le terrain. Deux types d'analyses complémentaires, quantitative et qualitative, ont été menées ; le protocole de recherche a reçu l'aval de la CNIL [6].

\section{1. La population d'étude}

Le recensement exhaustif des admissions indique que parmi 263 jeunes admis avant 2000 (incluant des accueils en urgence, des placements courts et des réorientations avant la fin de 1'adolescence), 150 étaient sortis de 1'institution à 15 ans et plus. Pour participer à l'étude, trois critères devaient être réunis: 1) avoir été placé au moins 3 ans, afin d'étudier les effets d'une prise en charge assez longue, 2) être sorti depuis 3 ans et plus, et 3) avoir au moins 23 ans à l'enquête; les deux derniers critères permettant d'avoir un recul suffisant par rapport aux événements qui entourent la sortie. L'étude 
concerne ainsi 130 sujets, avec des admissions entre 1963 et 1992 (certains jeunes avaient transité par un autre VE) et des sorties de 1972 à 2001. Aujourd'hui, ces personnes ont entre 23 et 50 ans (âge médian : 38 ans).

\subsection{Le recueil des données}

Une enquête postale à toute la population d'étude (123 anciens placés, sept sont décédés) a été suivie d'entretiens semi-directifs. Le taux de réponse est élevé puisque 99 anciens placés ont renvoyé leur questionnaire et 73 ont été rencontrés. Un matériel important a été obtenu par le biais de plusieurs sources: outre cette enquête, des informations ont été recueillies auprès des membres de l'équipe socio-éducative (22 mères SOS, 2 directeurs de VE) et dans les dossiers institutionnels. Le fait que cette institution dispose depuis longtemps de fiches de synthèse retraçant l'évolution annuelle de l'enfant (scolarité, observations du comportement, [7]...) a permis de retracer pour chacun d'eux la chronologie des événements importants de leur vie.

Les thématiques explorées dans cet article sont:

- La situation à l'admission : a) problématiques familiales, antécédents parentaux tels que les pathologies psychiatriques et sociales, alcoolisme...; b) âge d'arrivée et placements antérieurs, carences graves et maltraitances (physiques, sexuelles, psychologiques) ;

- Le séjour en placement VE : cursus scolaire avec ou sans retard, en filières adaptées (perfectionnement, CPPN, SES) ou médicalisées (IME, IMP) et formation professionnelle, modalités des prises en charge (aides scolaires, orthophonie, suivi psychologique...) et durée; troubles psychiques et comportementaux, relations avec les parents (non étudiés dans cet article).

- La fin des prises en charge et les aides mises en place.

\subsection{Analyses}


Le repérage et l'interprétation des problèmes psychologiques et comportementaux, à partir des dossiers individuels, ont été effectués par deux psychologues, extérieures à 1'institution. La variable «Troubles » fondée sur la co-occurrence des symptômes permet de les hiérarchiser en fonction de leur gravité (pas de troubles notoires mentionnés, troubles légers, troubles graves). Deux périodes ont été distinguées: à l'admission et au début du placement, puis à l'adolescence et la sortie.

Si la population d'étude recouvre 37 fratries, ce sont les antécédents parentaux et les facteurs de risque individuels qui ont été retenus dans les analyses.

Les différences significatives dans la comparaison des distributions ont été mises en évidence par des analyses bivariées; des analyses multivariées par régression logistique multiple ont dégagé le rôle propre de facteurs déterminants après ajustement sur les autres facteurs (logiciel SAS). Les variables sélectionnées pour être testées dans la régression logistique multiple sont celles manifestant une différence significative au seuil de 20 pour cent dans l'analyse bivariée. Le risque $\mathrm{OR}_{\mathrm{a}}$ mesure le coefficient multiplicateur du risque étudié.

\section{Résultats}

\subsection{Description des fratries accueillies et durée du placement}

Compte tenu de l'évolution dans le temps des motifs d'admission et des prises en charge, trois périodes historiques ont été distinguées : 1) la période la plus ancienne (Pa) avec des sujets âgés de 42-50 ans à l'enquête, nés entre 1953-1961 $(\mathrm{n}=31), 2)$ la période moyenne $(\mathrm{Pm})$ avec des sujets de 34 à 41 ans nés entre 1962-1969 $(\mathrm{n}=62)$, et 3$)$ la période la plus récente $(\mathrm{Pj})$ avec des sujets de moins de 34 ans et nés entre 1970-1982 
$(n=30)^{1}$. Le tableau 1 présente les principales caractéristiques des jeunes au moment de l'admission et au cours du placement en VE.

Tableau 1

Avec le temps, la taille des fratries accueillies a diminué : plus de la moitié des jeunes nés dans les années 50 ont été admis avec au moins 5 frères ou sœurs (d'où des âges d'entrée plus tardifs) ; ce n'est le cas que pour $10 \%$ des jeunes dans les années 70 . Ainsi, plusieurs fratries cohabitent dans la même maison dans la période $\mathrm{Pj}$; les enfants de cette génération, arrivés plus jeunes, vont rester plus longtemps en placement. Dans la moitié des cas et quelle que soit la période, ce village d'enfants a accueilli des fratries incomplètes (aînés placés ailleurs, restés en famille...). De plus, sur les 37 fratries prises en charge, sept regroupaient des frères et sœurs qui n'avaient pas vécu ensemble avant le placement. Les prises en charge ont été de longue durée : au moins treize ans pour un tiers des sujets (durée médiane : neuf ans).

\subsection{Facteurs de risque familiaux et maltraitances}

Avant l'admission en VE, les enfants ont vécu dans des contextes familiaux très précaires et violents, ayant fait l'objet de suivis sociaux dans les deux tiers des familles. Si plusieurs motifs étaient à l'origine du placement, le décès ou la disparition parentale en était la raison principale. Dans près d'une fratrie sur quatre, on dénombre des morts violentes (pour l'un ou les deux parents) et des problèmes de santé physique pour un tiers des parents. Les trois quarts des jeunes avaient au moins un parent concerné par l'alcool, plus fréquemment le père $(68 \%)$ mais aussi la mère $(25 \%) ; 30 \%$ une pathologie psychiatrique ( $15 \%$ chez le père, $17 \%$ chez la mère), et $32 \%$ avaient au moins un parent qui présentait une pathologie sociale (délinquance, prostitution...), à

\footnotetext{
${ }^{1}$ Le choix des bornes d'âge se justifie par un meilleur équilibrage des tranches d'âge extrêmes.
} 
égalité le père et la mère (22\%). Avec le temps, entre $\mathrm{Pa}$ et $\mathrm{Pj}$, la nature des antécédents parentaux, mieux repérés, se transforme: si l'alcoolisme diminue, certains antécédants maternels sont plus élevés : la pathologie psychiatrique de la mère passe de $2 \%$ à $62 \%$, et la délinquance maternelle de $2 \%$ à $48 \%$.

Quelle que soit la génération, les deux tiers des enfants ont été victimes de maltraitances, allant de carences et négligences graves aux mauvais traitements ( 7 dans des placements antérieurs; 11 situations d'abus sexuels concernant parfois plusieurs membres d'une fratrie). Quatre sur cinq ont été placés antérieurement, dont $22 \%$ deux ans et plus. La gravité des maltraitances augmente en fonction des dysfonctionnements familiaux, et conduit à des risques plus élevés de placement avant l'arrivée au VE.

Les facteurs de risque familiaux se cumulant, des analyses multivariées ont été effectuées, tenant compte aussi de l'âge et du sexe. Une première analyse prenant en compte les facteurs caractéristiques des pathologies parentales (père et/ou mère) montre que trois facteurs ont un rôle déterminant dans la survenue des violences conjugales : la délinquance maternelle $\left(\mathrm{OR}_{\mathrm{a}}=8,69\right)$, l'alcoolisme et la pathologie psychiatrique du père (respectivement $\mathrm{OR}_{\mathrm{a}}=9,64$ et $\mathrm{OR}_{\mathrm{a}}=7,01$ ). De même, une autre analyse met en évidence que les mauvais traitements sont plus fréquents en cas d'alcoolisme de la mère $\left(\mathrm{OR}_{\mathrm{a}}=2,86\right)$ et en cas de pathologie sociale du père $\left(\mathrm{OR}_{\mathrm{a}}=3,41\right)$. Ces deux facteurs jouent indépendamment l'un de l'autre. Le pourcentage d'enfants maltraités est élevé en cas d'alcoolisme maternel et/ou de pathologie sociale paternelle (de 50 à $62 \%$ ) ; en 1'absence de ces deux pathologies, cette proportion est de $24 \%$.

Par ailleurs, près de quatre jeunes sur dix présentaient des difficultés de santé (pathologies et handicaps divers) et des retards importants de développement physique et intellectuel au moment de leur arrivée en placement. 


\subsection{Scolarité et prises en charge pendant le placement}

En raison de leur âge, un quart des jeunes n'étaient pas encore scolarisés à l'admission ; pour les autres, les retards scolaires sont déjà importants : $18 \%$ d'entre eux ont plus de deux ans de retard ou sont en filières adaptées (perfectionnement, SES...), voire médicalisées (11 enfants). Lors de l'entrée en $6^{\text {ème }}, 7 \%$ des jeunes sont en filières médicalisées et $42 \%$ sont en échec scolaire grave ( 3 ans de retard et/ou filières adaptées de 1'Education nationale). Ces échecs scolaires graves varient selon l'âge d'admission et sont les plus fréquents pour ceux arrivés entre 8 et 10 ans $(68 \%)$, ces jeunes n'ayant pas pu (encore) rattraper leur retard acquis auparavant.

Les rééducations et suivis psycho-éducatifs réguliers et supérieurs à un an (aides scolaires, psychomotricité, orthophonie, suivi psychologique et/ou thérapeutique hors VE en CMP, CMPP...) ont concerné 53\% des enfants et adolescents. Ces prises en charge se sont diversifiées et amplifiées avec le temps : 83\% en ont bénéficié dans la plus jeune génération vs $17 \%$ pour les plus anciens. Les suivis thérapeutiques réguliers hors VE concernent $38 \%$ des enfants, deux fois plus souvent les enfants atteints de troubles graves. Ce sont essentiellement les plus jeunes générations qui en ont bénéficié $(76 \%)$. Il en est de même pour les aides au moment de la sortie ${ }^{2}$.

\subsection{Troubles psychologiques et comportementaux}

Les difficultés vécues dans l'enfance et les souffrances associées expliquent l'importance des troubles des enfants.

\subsubsection{Evolution des troubles}

Si pour 35 jeunes, un peu plus de filles que de garçons, aucun trouble notoire n'a été mentionné, pour 4 autres (dont 3 admis à 10 ans et plus), des problèmes sont soit

\footnotetext{
${ }^{2}$ Un article ultérieur explorera la problématique de la sortie du placement et de 1'entrée dans l'âge adulte.
} 
apparus au cours du placement (à moins qu'ils n'aient pas été repérés et notés lors de 1'admission), soit se sont amplifiés (opposition à la mère SOS).

Tableau 2

Pour 34 jeunes, les troubles, troubles légers dans 8 cas sur 10 (souvent liés aux environnements précédant l'admission et/ou à l'adaptation au nouveau lieu de vie) se sont estompés ou ont disparu. Il s'agit en premier lieu de troubles psychosomatiques (énurésie, terreurs nocturnes) et de symptomatologie anxieuse, puis de troubles du contrôle pulsionnel (instabilité, impulsivité, problèmes caractériels). Enfin, viennent les difficultés relationnelles avec la mère SOS, en proportion moindre.

Enfin, les difficultés se sont maintenues pour 46 sujets $(50 \%$ de troubles graves, en majorité des garçons). On dénombre autant de troubles d'ordre anti-social (violences, agressivité, fugues, impulsivité, actes de délinquance, forte consommation d'alcool) que du registre de l'«introversion» (inhibitions, repli sur soi, dépression...). Dans la moitié des cas, ce sont surtout les problèmes relationnels qui sont mentionnés: oppositions parfois très violentes de la part des jeunes mais aussi rejets - surtout à l'adolescence - de la part de certaines mères SOS rigides. Ceci pourrait expliquer en partie que les troubles de comportement concernent davantage certaines fratries que d'autres. Le nombre de fratries élevées ensemble peut également influer : par exemple, dans deux situations où des fratries «difficiles» ont été élevées ensemble, dans la première $4 / 7$ jeunes présentaient des troubles importants et 4/6 dans 1'autre.

Etant donné la très forte corrélation des troubles entre les deux périodes (début du placement, adolescence et sortie), ils ont été regroupés en une seule variable qui rend compte de l'ensemble des problèmes sur la durée du placement. Quelle que soit l'appartenance générationnelle, ce sont environ sept jeunes sur dix qui ont eu des troubles pendant le placement. Parmi ces jeunes, quatre sur dix en ont encore à 
l'adolescence et au moment de la sortie (troubles graves pour plus de la moitié d'entre eux).

Tableau 3

Deux facteurs sont liés à la persistance ou l'aggravation de troubles : 1'âge d'arrivée à six ans et plus et le nombre de placements antérieurs (Tableau 3). Toutefois, ceux arrivés jeunes (avant l'âge de 6 ans) dont les troubles persistent ont tous eu des placements antérieurs.

3.3.2. Impact des facteurs de risque

Les analyses ont montré la nature spécifique de certains facteurs de risque liés aux problèmes psychologiques et comportementaux des jeunes (Tableau 4A).

Tableau 4A

Ainsi, qu'ils soient légers ou graves, les troubles sont plus fréquemment observés en cas de pathologie psychiatrique de l'un ou des deux parents. Par ailleurs, les troubles graves sont deux fois plus importants en cas de mauvais traitements que dans les autres situations (carences, négligences...).

\section{Tableau 4B}

La prise en compte simultanée de ces facteurs met en évidence que trois d'entre eux conservent un rôle propre face à la gravité des troubles (tableau 4B): avoir subi des mauvais traitements $\left(\mathrm{OR}_{\mathrm{a}}=3,94\right)$, avoir un ou deux parents présentant une pathologie psychiatrique $\left(\mathrm{OR}_{\mathrm{a}}=2,60\right)$, et être en échec scolaire grave ou suivre une filière adaptée ou médicalisée $\left(\mathrm{OR}_{\mathrm{a}}=2,26\right)$ augmente le risque de manifestations de troubles psychologiques et comportementaux graves.

\section{Discussion}


A la différence des autres institutions de placement dans le cadre de la Protection de 1'enfance, les villages d'enfants SOS offrent des accueils de longue durée pour des fratries. La qualité des dossiers archivés depuis trente années a permis d'analyser l'évolution des prises en charge. L'étude montre un développement et une diversification des aides et des suivis, notamment thérapeutiques, auprès des jeunes placés. En témoignent ces deux extraits d'entretiens illustrant cette évolution. Le premier est celui d'une femme aujourd'hui âgée de 40 d'ans qui se souvient n'avoir rencontré que très ponctuellement la psychologue du VE. Elle estime que les problèmes au sein de sa fratrie ne pouvaient être gérés par celle qui les a élevés : "Nous mettre dans une famille, c'est bien mais faut aller plus loin que ça, et on n'est pas allé plus loin que ça. Je pense qu'on n'aurait pas une vie de famille comme on a aujourd'hui, à se déchirer les uns les autres ou à continuer à boire... Il faut creuser les problèmes chez l'enfant». Le second est celui d'un homme d'environ 35 ans : "J'ai eu un soutien psychologique depuis tout petit. Ca m'a aidé. Au départ j'étais par partant [...] A l'adolescence, au début j'y allais avec cour, et après moins parce que j'avais plus envie d'aller voir mes copains et mes copines. C'est devenu assez irrégulier... En tout cas, si j'avais pas eu ça, je sais pas ce que je serais devemu. " Ces constats rejoignent ceux de l'étude menée avec la Fondation Grancher qui avaient montré l'importance des soins et accompagnements réalisés par les équipes lors de la transformation de l'institution en placement familial spécialisé [8].

Concernant les troubles psychiques, des précautions méthodologiques et interprétatives s'imposent. Les données recueillies restent descriptives et pour certaines approximatives sur le plan de la terminologie et partielles quant à l'évaluation clinique [9]. Cependant, l'utilisation de grilles évaluatives standardisées a rarement été utilisée dans les études françaises sur les enfants placés [2, 10]. 
L'interprétation des résultats reste complexe compte tenu de la longue durée observée (30 années) pendant laquelle les profils d'admission se sont modifiés. Ce sont surtout les troubles les plus bruyants qui ont été davantage repérés, la gravité de certaines manifestations de souffrances psychiques est restée inaperçue. Le retentissement des maltraitances graves sur le développement psychoaffectif et intellectuel est resté longtemps méconnu et ce type de placement n'y échappe pas : professionnels et mères SOS ont longtemps cru qu' «avec le temps, les choses s'arrangeraient». Il serait intéressant, par ailleurs, d'étudier les interactions entre les jeunes et les mères SOS qui les ont élevés.

Des analyses approfondies ont mis l'accent sur le rôle propre de certains facteurs déterminants : 1'impact des violences intra-familiales, des maltraitances et de certaines pathologies parentales sur les troubles psychiques des jeunes $[11,12,13,14]$. Les résultats soulignent aussi les répercussions majeures de ces troubles sur les apprentissages et le cursus scolaire [15]

Ces prises en charge en village d'enfants ont permis une amélioration de la situation comportementale et scolaire des jeunes [16]. Toutefois, un tiers de ceux qui présentaient des troubles graves (la moitié en cas de troubles légers) n'ont pas eu de suivi thérapeutique régulier. En parallèle, ces troubles graves concernent tout autant les anciennes générations accueillies que les plus récentes, alors que ces dernières ont davantage bénéficié de suivis thérapeutiques spécifiques hors VE. Ceci conduit à penser qu'on assiste à un effet de seuil : face à un certain niveau de gravité des souffrances psychiques, ce placement en village d'enfants, même stable et de longue durée, n'est pas un placement thérapeutique. Le travail clinique en protection de l'enfance est une véritable spécialité [17]. En outre, avec cette étude, la pratique du regroupement de plusieurs fratries peut être questionnée au regard du risque d'amplification de ces 
troubles chez des enfants élevés avec d'autres jeunes très perturbés. C'est la question que se pose Régine Scelles, dans une étude récente menée auprès des professionnels de villages d'enfants, qui souligne la nécessité de ne pas réunir des fratries présentant « les mêmes pathologies du lien » [18].

\section{Pour conclure}

$\mathrm{Au}$ final, les transformations intervenues pendant ces trois dernières décennies traduisent les changements profonds dans la vie des enfants placés, sous l'impulsion de pionniers tels que Michel Soulé et Myriam David. Cette évolution se poursuit aujourd'hui encore, les besoins psychoaffectifs des enfants sont mieux pris en compte par la loi du 5 mars 2007 réformant la Protection de l'enfance. Ainsi, l'enfant accueilli au-delà d'une durée supérieure à deux ans doit pouvoir bénéficier d'une «continuité relationnelle, affective, géographique dans son lieu de vie».

Enfin, au regard de l'impact des problèmes de santé mentale sur l'insertion socioprofessionnelle et la vie relationnelle à moyen et long terme, la question de l'évaluation clinique des problématiques psychiques individuelles devient aujourd'hui incontournable. Leur repérage doit avoir lieu dès 1'admission en placement ou dans les mois qui suivent pour mettre en place le plus tôt possible des soins adaptés sans les démultiplier, dans le cas d'une prise en charge qui articule l'éducatif et le thérapeutique.

"La méconnaissance de la nature des troubles qui sont à l'origine des placements au long cours et de leurs conséquences sur le déroulement du placement est le point central et crucial auquel on se heurte... " M. David, 2004 p. 443 [19] 


\section{Remerciements :}

Le programme de recherche a été financé en premier lieu par 1'Association SOS Villages d'Enfants et a reçu une subvention de la Fondation de France. Il a bénéficié de l'infrastructure et des ressources du CERMES-Inserm U750. Les auteurs remercient également Marie Constantin-Kuntz et Catherine Mousseau-Stella, psychologues qui ont participé à l'analyse des dossiers et aux entretiens, ainsi que Roselyne Stella, secrétaire au village d'enfants de Marseille qui a contribué activement à l'enquête postale. 
Table 1 A : Principales caractéristiques à l'admission selon les générations (\% colonnes)

\begin{tabular}{|c|c|c|c|c|c|}
\hline \multirow[b]{2}{*}{ 1A. A l'admission } & \multicolumn{2}{|c|}{ Total } & \multicolumn{3}{|c|}{ Age actuel } \\
\hline & $N=123$ & $(\%)$ & $\begin{array}{c}\geq 42 \text { ans } \\
\left(\begin{array}{c}\mathrm{Pa}, \mathrm{n}=31) \\
\%\end{array}\right. \\
\end{array}$ & $\begin{array}{c}34-41 \text { ans } \\
\left(\begin{array}{c}P m, n=62) \\
\%\end{array}\right.\end{array}$ & $\begin{array}{c}\leq 33 \text { ans } \\
(\mathrm{Pj}, \mathrm{n}=30) \\
\%\end{array}$ \\
\hline \multicolumn{6}{|l|}{ Genre } \\
\hline masculin & 61 & 50 & 42 & 52 & 53 \\
\hline féminin & 62 & 50 & 58 & 48 & 47 \\
\hline \multicolumn{6}{|l|}{$\mathrm{Nb}$ enfants de la fratrie accueillis en VE } \\
\hline $1-2$ & 21 & 17 & 10 & 10 & 40 \\
\hline 3 & 24 & 20 & 16 & 21 & 20 \\
\hline $4-5$ & 34 & 28 & 16 & 32 & 30 \\
\hline \multirow[t]{2}{*}{$6-10$} & 44 & 36 & 58 & 37 & 10 \\
\hline & & & & $p<0,001$ & \\
\hline \multicolumn{6}{|l|}{$\mathrm{Nb}$ de fratries vivant ensemble } \\
\hline $1-2$ & 86 & 70 & 74 & 77 & 50 \\
\hline \multirow[t]{2}{*}{3} & 37 & 30 & 26 & 23 & 50 \\
\hline & & & & $p<0,03$ & \\
\hline \multicolumn{6}{|l|}{ Age à l'entrée en VE } \\
\hline $0-5$ ans & 31 & 25 & 6 & 26 & 43 \\
\hline $6-7$ & 21 & 17 & 13 & 16 & 23 \\
\hline $8-10$ ans & 32 & 26 & 29 & 29 & 17 \\
\hline \multirow{2}{*}{$11-16$ ans } & 39 & 32 & 52 & 29 & 17 \\
\hline & & & & $p<0,01$ & \\
\hline \multicolumn{6}{|l|}{ Placement antérieur au VE } \\
\hline aucun & 24 & 20 & 26 & 16 & 20 \\
\hline 1 seul & 70 & 57 & 61 & 56 & 53 \\
\hline$\geq 2$ placements antérieurs & 29 & 24 & 13 & 27 & 27 \\
\hline \multicolumn{6}{|l|}{ Durée totale des placements antérieurs } \\
\hline 0-3 mois & 46 & 39 & 35 & 37 & 48 \\
\hline 4-12 mois & 45 & 38 & 48 & 40 & 23 \\
\hline$\geq 2$ années & 26 & 22 & 16 & 23 & 28 \\
\hline \multicolumn{6}{|l|}{ Pathologie sociale de la mère et/ou du père } \\
\hline non & 81 & 68 & 81 & 66 & 57 \\
\hline oui & 39 & 32 & 19 & 34 & 43 \\
\hline \multicolumn{6}{|l|}{ Alcoolisme de la mère et/ou du père } \\
\hline non & 31 & 26 & 13 & 23 & 45 \\
\hline \multirow{2}{*}{ oui } & 90 & 74 & 87 & 77 & 55 \\
\hline & & & & $p<0,02$ & \\
\hline \multicolumn{6}{|c|}{ Pathologie psychiatrique de la mère et/ou du père } \\
\hline non & 86 & 70 & 90 & 77 & 33 \\
\hline \multirow[t]{2}{*}{ oui } & 37 & 30 & 10 & 23 & 67 \\
\hline & & & & $p<0,001$ & \\
\hline \multicolumn{6}{|l|}{ Violences intra-conjugales } \\
\hline non & 41 & 35 & 32 & 26 & 55 \\
\hline \multirow[t]{2}{*}{ oui } & 77 & 65 & 68 & 74 & 45 \\
\hline & & & & $p<0,03$ & \\
\hline \multicolumn{6}{|l|}{ Négligences graves, mauvais traitements (MT) } \\
\hline non & 43 & 35 & 35 & 31 & 43 \\
\hline carences, négligences graves (sans MT) & 36 & 29 & 26 & 34 & 23 \\
\hline mauvais traitements & 44 & 36 & 39 & 35 & 33 \\
\hline
\end{tabular}

Note : seules les différences significatives au seuil $\mathrm{p}<0,05$ ont été mentionnées 
Table 1 B : Principales caractéristiques pendant le placement selon les générations (\% colonnes)

\begin{tabular}{|c|c|c|c|c|c|}
\hline \multirow[t]{2}{*}{ 1.B. Pendant le placement en VE } & \multicolumn{2}{|c|}{ Total } & \multirow{2}{*}{\multicolumn{2}{|c|}{$\begin{array}{c}\text { Age actuel } \\
\text { 34-41 ans } \\
\left(\begin{array}{c}\mathrm{Pm}, \mathrm{n}=62) \\
\%\end{array}\right.\end{array}$}} & \multirow{2}{*}{$\begin{array}{c}\leq \mathbf{3 3} \text { ans } \\
(\mathrm{Pj}, \mathbf{n}=\mathbf{3 0}) \\
\%\end{array}$} \\
\hline & $N=123$ & $\%$ & & & \\
\hline \multicolumn{6}{|l|}{ Retard scolaire à l'admission (>maternelle) } \\
\hline aucun retard ou 1 an & 44 & 47 & 45 & 44 & 59 \\
\hline 2 ans & 33 & 35 & 41 & 35 & 24 \\
\hline 3 ans (yc filières adaptées ou médicalisées) & 17 & 18 & 14 & 21 & 18 \\
\hline \multicolumn{6}{|l|}{ Scolarité à l'entrée en 6ème } \\
\hline aucun retard, ou 1 an de retard & 44 & 36 & 29 & 24 & 67 \\
\hline 2 ans de retard & 19 & 15 & 13 & 19 & 10 \\
\hline 3 ans (yc filières adaptées ou médicalisées) & 60 & 49 & 58 & $\begin{array}{c}56 \\
p<0,01\end{array}$ & 23 \\
\hline \multicolumn{6}{|l|}{ Troubles psychologiques et comportementaux } \\
\hline aucun & 35 & 28 & 39 & 27 & 20 \\
\hline légers & 37 & 30 & 29 & 27 & 37 \\
\hline graves & 51 & 41 & 32 & 45 & 43 \\
\hline \multicolumn{6}{|l|}{ Prises en charge psycho-éducatives } \\
\hline aucune ou ponctuelles seulement & 56 & 47 & 83 & 45 & 17 \\
\hline régulières (avec suivi $\geq 1$ an) & 62 & 53 & 17 & $\begin{array}{c}55 \\
p<0,001\end{array}$ & 83 \\
\hline \multicolumn{6}{|l|}{ Suivi psychologique extérieur au VE (CMPP...) } \\
\hline non & 79 & 68 & 92 & 66 & 24 \\
\hline oui & 38 & 32 & 8 & $\begin{array}{c}34 \\
p<0,001\end{array}$ & 76 \\
\hline \multicolumn{6}{|l|}{ Durée de la prise en charge } \\
\hline$\leq 5$ ans & 27 & 22 & 42 & 19 & 7 \\
\hline $6-8$ ans & 34 & 28 & 32 & 31 & 17 \\
\hline $9-12$ ans & 28 & 23 & 19 & 24 & 23 \\
\hline 13 ans & 34 & 28 & 6 & $\begin{array}{c}26 \\
p<0,001\end{array}$ & 53 \\
\hline
\end{tabular}


Tableau 2 : Troubles pendant le placement (effectifs)

\begin{tabular}{clll}
\hline $\begin{array}{c}\text { Troubles } \rightarrow \\
\downarrow \text { Début du placement }\end{array}$ & \multicolumn{2}{c}{ Adolescence et sortie } & $\begin{array}{c}\text { Total } \\
\text { (n) }\end{array}$ \\
& non & oui & \\
\hline non & $35(13 G, 22 F)$ & $4(3 G, 1 F)$ & 39 \\
oui & $34(16 G, 18 F)$ & $46(27 G, 19 F)$ & 80 \\
\hline Total (n) & 69 & 50 & 119 \\
\hline \multicolumn{2}{c}{ Note: $\mathrm{G}:$ garçon, $\mathrm{F}:$ fille } & &
\end{tabular}

Table 3 : Evolution des troubles selon l'âge à l'admission (\% lignes)

\begin{tabular}{cccccc}
\hline $\begin{array}{c}\text { Age à } \\
\text { l'admission }\end{array}$ & aucun & $\begin{array}{c}\text { Evolution des troubles } \\
\text { disparition }\end{array}$ & diminution & persistance & Total $(\boldsymbol{n})$ \\
\hline $0-5$ ans & 17 & 34 & 28 & 21 & 29 \\
$6-7$ ans & 20 & 30 & 15 & 35 & 20 \\
$8-10$ ans & 35 & 26 & 6 & 32 & 31 \\
$11-15$ ans & 38 & 26 & 8 & 28 & $\mathbf{3 9}$ \\
\hline
\end{tabular}


Tableau 4A : Facteurs de risque et troubles (\% lignes)

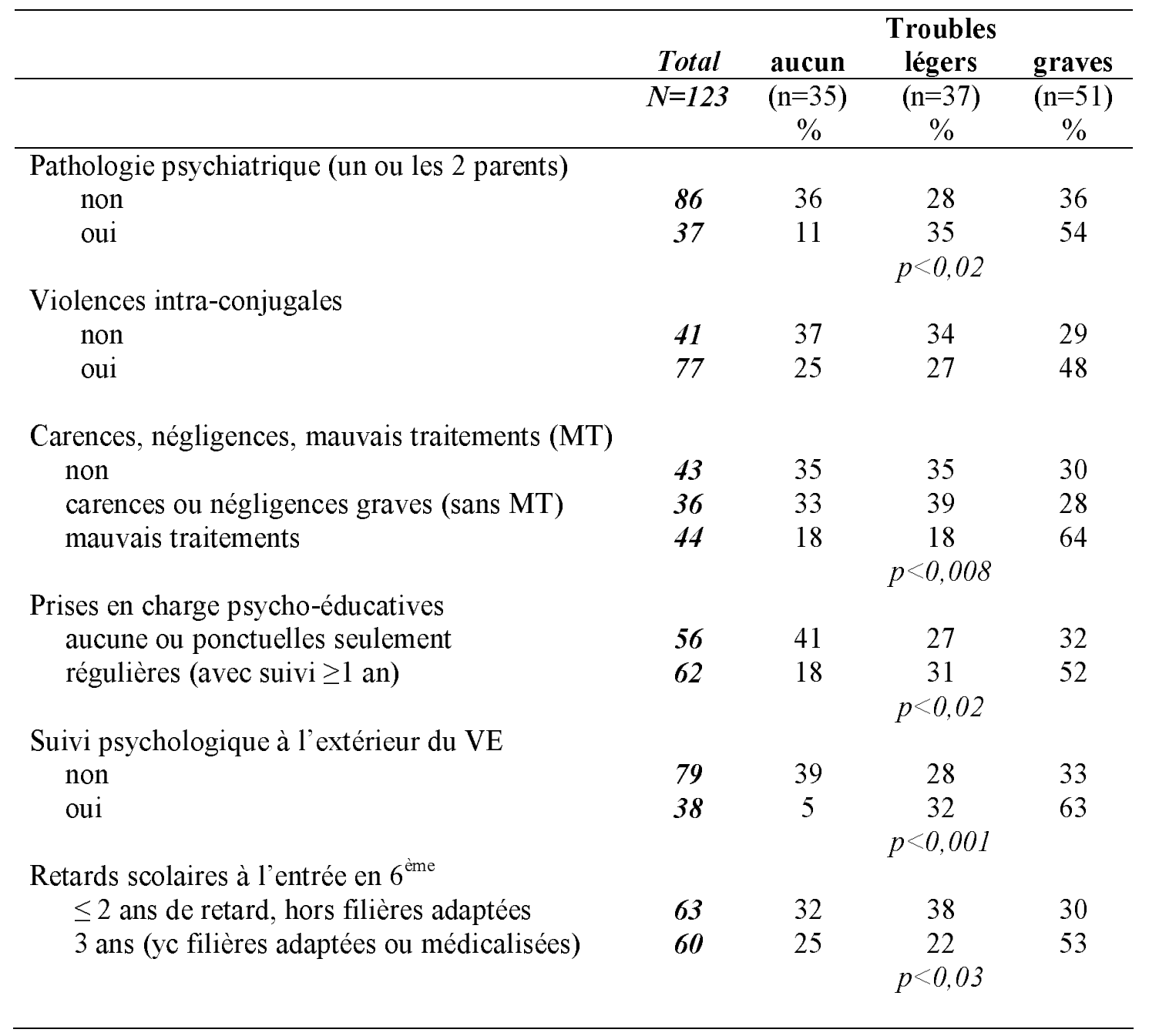


Tableau 4B : Facteurs de risque et troubles graves

(régression logistique multiple)

\begin{tabular}{lcccc}
\hline & \multicolumn{5}{c}{ Troubles graves } \\
& $\boldsymbol{n}$ & $\mathrm{OR}_{\mathrm{b}}$ & $\mathrm{OR}_{\mathrm{a}}$ & $\mathrm{p}$ \\
\hline Carences et/ou mauvais traitements & $\mathbf{4 3}$ & - & - & \\
$\quad$ non & $\mathbf{3 6}$ & 0,89 & 1,06 & \\
$\quad$ carences sans MT & $\mathbf{4 4}$ & 4,04 & 3,94 & 0,007 \\
$\quad$ mauvais traitements & $\mathbf{8 6}$ & - & - & \\
Pathologie psychiatrique (un ou les 2 parents) & $\mathbf{3 7}$ & 2,09 & 2,60 & 0,04 \\
$\quad$ non & & & & \\
$\quad$ oui & $\mathbf{6 3}$ & - & - & \\
Retard scolaire à l'entrée en 6ème & $\mathbf{6 0}$ & 2,65 & 2,26 & 0,05 \\
$\quad$ 0-2 ans & & & & \\
3 ans, ou en filières adaptée ou médicalisée &
\end{tabular}

$\mathrm{ORb}$ : Odds-ratio brut, $\mathrm{ORa}:$ Odds-ratio ajusté.

Ex. de lecture : 3,94 indique un risque près de 4 fois plus élevé de troubles graves en cas de mauvais traitements, indépendamment des deux autres facteurs significatifs (pathologie psychiatrique et retard scolaire grave) 


\section{Références}

1 Naves P., Briand C., Oui A. Pour et avec les enfants et adolescents, leurs parents et les professionnels. Contribution à l'amélioration du système français de Protection de l'Enfance. Rapport du groupe de travail Protection de 1'enfance et de l'adolescence au Ministre délégué à la Famille, 2003.

2 Boutanquoi M., Minary JP. Demiche T. La qualité des pratiques en protection de l'enfance. Rapport à la DGAS, Ministère de la Santé et des Solidarités, 2005.

3 Berger M. L'échec de la protection de l'enfance, Paris: Dunod; 2004.

4 Ministère de 1'Emploi, de la Cohésion sociale et du Logement, Ministère de la Santé et des Solidarités : Livret d'information pour les acteurs de la Protection de l'Enfance. La qualité et son évaluation, 2006.

5 Frechon I., Dumaret A.C. Bilan critique de 50 ans d'études sur le devenir des enfants placés. Neuropsychiatr Enfance Adolesc 2008;56:135-147.

6 Dumaret A.C., Constantin-Kuntz M., Crost M. Devenir à 1'âge adulte des jeunes placés avec leur fratrie au Village d'Enfants de Marseille. Rapport de recherche à 1'Association SOS Villages d'Enfants, Villejuif, INSERM U750-CERMES, 2006.

7 Dumaret A., Duyme M. Devenir scolaire et professionnel de sujets placés en villages d'enfants. Rev Int Psychol Appl 1982;4:455-474.

8 Coppel M. Dumaret A.C. Que sont-ils devenus ? Les enfants placés à 1'CEuvre Grancher. Analyse d'un placement spécialisé. Ramonville Saint-Agne: Erès; 1995.

9 Bursztejn C. Les troubles des conduites: abord nosologique et épistémologique. Neuropsychiatr Enfance Adolesc 2007;55:443-449.

10 Dumaret A.C., Ruffin D. (1999) Bilan socio-scolaire et prises en charge des jeunes accueillis en placement familial. Comportements et perceptions des adultes. Rapport de recherche (Convention d'étude INSERM-SLEA, Lyon). On line : http:/oned.gouv.fr/

11 Becker de E. L'impact des violences conjugales sur les mineurs d'âge. Neuropsychiatr Enf Adol 2008;56:21-26.

12 Cahill L.T., Kaminer R.K., Johnson P.G. Developmental, cognitive and behavioral sequelae of child abuse. Child Adolesc Psychiatric Clin N America 1999;8:827-843.

13 Svedin, G., Wadsby, M. and Sydsjö, G. Children of mothers who are at psychosocial risk. Mental health, behavior problems and incidence of child abuse at 8 years. European Child and Adolescent Psychiatry 1996;5: 162-71.

14 Kitzmann, K.M., Gaylord, N.K., Holt, A.R., Kenny, E.D. Child witnesses to 
domestic violence: a meta-analytic review. Journal of Consulting and Clinical Psychology 2003;71:339-352.

15 Kendall-Tackett, K.A., Eckenrode, J. The effect of neglect on academic and disciplinary problems: a developmental perspective. Child Abuse Neglect 1996:20:161169.

16 Horwitz, S.McCue, Balestracci K.M., Simms M.D. Foster care placement improves children's functioning. Arch Pediatr Adol Med 2001;155:1255-1260.

17 Berger, M. La spécificité du travail clinique en protection de l'enfance. Neuropsychiatr Enf Adol 2006;24: 137-141.

18 Scelles, R. Accueil conjoint des enfants d'une même fratrie dans le cas de placement: intérêts et limites. Revue Internationale d'Education Familiale 2006;2:7983.

19 David M. Le placement familial. Paris: ESF (1989) 2è ed 2004. 